\title{
Functional outcomes in early (T1/T2) supraglottic cancer: a systematic review
}

\author{
Benjamin van der Woerd ${ }^{1} \mathbb{B}$, Krupal B. Patel ${ }^{2}$, Anthony C. Nichols ${ }^{1}$, Kevin Fung ${ }^{1}$, John Yoo ${ }^{1}$ and S. Danielle MacNeil ${ }^{*}$
}

\begin{abstract}
Objectives: Organ preserving surgery (OPS) and radiotherapy (RT) are both accepted treatment options for early stage supraglottic cancer (SGC). Radiation has supplanted surgery in most cases, because of the perception that surgery results in poorer functional outcomes. However, evidence suggests that OPS with a neck dissection may be associated with improved survival. Our objective was to conduct a systematic review of the literature to compare functional outcomes of OPS and RT for early SGC.

Methods: We searched Medline, EMBASE and Cochrane Central Register of Controlled Trials to identify studies. Studies were included if they reported functional outcomes on 10 or more patients with early stage SGC treated with radiation or OPS, including open partial laryngectomy, transoral laser microsurgery (TLM) or transoral robotic surgery (TORS). Two reviewers independently screened articles for relevance using pre-determined criteria.

Results: From 7720 references, we included 10 articles ( $n=640$ patients). 50\% ( $n=320$ ) of patients were treated with surgery. Three head-to-head RT versus OPS papers were included, however different outcome measures were used for each group. Intractable aspiration management (including total laryngectomy or permanent tracheostomy) following OPS was reported in five papers representing 186 patients; the definitive intractable aspiration management rate was $2.6 \%$ (95\% Cl 1.0-6.8\%). Four papers reported permanent G-tube rate for the surgical group ( $n=198)$, calculating a rate of $5.3 \%(95 \% \mathrm{Cl} 2.6-10.5 \%)$, this was not reported for the RT group in any papers. One study reported quality of life. Two studies reported objective voice measures.

Conclusions: This systematic review revealed a paucity of objective measures and significant data heterogeneity, rendering the comparison of functional outcomes following OPS versus RT for early SGC limited. Future research should include objective measures of functional outcomes including laryngectomy rate, g-tube rate, tracheostomy dependence, quality of life, and voice quality measures.
\end{abstract}

Keywords: Early stage, Supraglottic squamous cell carcinoma, Supraglottic SCC, Outcomes, Systematic review, Functional outcomes

\section{Introduction}

Early stage supraglottic squamous cell carcinoma (SSCC) includes T1 tumours, isolated to one subsite of the supraglottis with normal vocal cord function, or T2 tumours, involving more than one subsite of the supraglottis, glottis, or surrounding tissue, without evidence of regional disease spread [1]. A recent study of nearly 160,000 laryngeal cancer SCC patients in the United States, found the incidence of SSCC to account for one third of laryngeal cancers [2].

\footnotetext{
* Correspondence: Danielle.MacNeil@|hsc.on.ca

${ }^{1}$ Department of Otolaryngology, Head \& Neck Surgery, Schulich Medicine \& Dentistry, Western University, London Health Sciences Centre, Victoria Hospital, London, Ontario, Canada

Full list of author information is available at the end of the article
}

Laryngeal cancers are the most common malignant lesions of the head and neck, with an estimated 13,150 new laryngeal cancers per year [3].

The recommendations from the National Comprehensive Cancer Network (NCCN) guidelines for treatment of early stage SSCC include both organ preservation strategies - radiation (RT) or organ preservation surgery (OPS) with or without a neck dissection [3, 4]. Despite small tumour sizes, 5-year survival for early stage SSCC is $64 \%$, and oncologic outcomes have not improved over the past 30 years $[2,5-8]$. This is hypothesized to be a result of the robust lymphatic supply to the supraglottis leading to higher rates of occult metastases to regional and distant

(c) The Author(s). 2018 Open Access This article is distributed under the terms of the Creative Commons Attribution 4.0 International License (http://creativecommons.org/licenses/by/4.0/), which permits unrestricted use, distribution, and 
sites. When comparing the relative 5-year survival from 1985 to 1987 to $1994-1996$, there was a decline from 52.2 to $47.3 \%$. Reviewing the data from the National Cancer Database, the largest decline was identified in patients with T1 N0-T2 N0 disease. A recent meta-analysis by Patel et al. (2018) examining survival in early stage supraglottic SCC suggested that primary surgery may result in decreased disease specific survival (OR 0.43, 95\% CI $0.31-0.60)$ and overall mortality (OR 0.40, 0.29-0.55) when compared with primary radiotherapy [9].

There are a limited number of studies, with no previous meta-analysis, that have compared the functional outcomes between primary surgery and primary radiotherapy for early stage SSCC. Much of the available data focuses on survival outcomes for the two treatment modalities [7-17]. There are no prospective clinical trials, and the majority of the studies reported are small and retrospective in design. Our objectives were to systematically review the literature to find all the relevant studies about the functional outcomes for surgery and radiation for early stage SSSC, synthesize the results and perform meta-analyses where possible.

\section{Methods}

A systematic review protocol was developed a priori to ensure the objectives and aims were outlined from the outset.

Computerized bibliographic databases: Medline, EMBASE and Cochrane Central Register of Controlled Trials were searched to identify studies. English language records were included from January 1990 to October 2018. The search strategy was designed by three authors (B.V.W., K.B.P. and S.D.M.) and an experienced librarian.

Randomized controlled trials, head-to-head comparative studies, observational studies, and case series that included 10 or more patients were reviewed. Single arm studies that reported functional results of organ preservation surgeries or radiotherapy as single modality therapy were included in the review. Studies on organ preserving surgeries, including open partial laryngectomy, transoral laser microsurgery (TLM) or transoral robotic surgery (TORS), with and without neck dissection were included.

The study population was limited to patients aged 18 and older, diagnosed with early stage supraglottic SCC (Tis, T1 N0, T2 N0). We excluded studies where functional results for patients with advanced $\mathrm{T}$ stage or node positive disease were grouped into the results and could not be reliably differentiated. We also excluded studies where functional outcomes for patients with early supraglottic cancers were combined with early glottic cancers.

Included studies were assessed for the following functional outcomes: aspiration; gastrostomy tube dependence; objective voice outcomes; and quality of life measures.

Titles, abstracts, and full texts of the studies were reviewed independently by two authors (B.V.W. and
K.B.P.). Disagreements were resolved by consensus. Inter-observer agreement was analyzed with Cohen's kappa. Titles were screened for the keywords: "squamous cell carcinoma" and "supraglottic", or "supraglottis", or "glottic", or "glottis", or "larynx", or "laryngeal". All study abstracts that met the eligibility criteria were then screened individually. The full text of studies that met inclusion criteria were then reviewed. The Newcastle-Ottawa Quality Assessment Scale for Cohort Studies was used to determine the quality of the studies (Table 2) [18]. Relevant data was extracted using a standardized data extraction form. Not all studies contained data for each of the outcome measures.

Review Manager 5.3 and Comprehensive Meta-Analysis applications were used for statistical analysis. Dichotomous outcomes were compared using odds ratios (OR) or weighted incidence rates and 95\% confidence intervals (CI). Heterogeneity across the studies was evaluated by the chi-square statistic and significance was set at $p<0.05$. The $\mathrm{I}^{2}$ test was used to measure the extent of inconsistency across the results.

A random effects model was used to allow for differences in the treatment effects from study to study. The $\mathrm{Z}$ statistic was used to test for overall pooled effect and significance was set at $\mathrm{p}<0.05$.

\section{Results}

The search strategy produced 7720 records. After duplicate records were removed from the search, 5218 unique records remained. After reviewing the titles, 1187 abstracts were deemed appropriate for abstract review. Following abstract review, 115 studies were appropriate for full text review. Ten studies met the final inclusion criteria after reviewing the full text. CONCLUSIONS:

There is an overall paucity of literature available regarding functional outcomes in the treatment of early stage SSCC patients, the majority of which is moderate in overall quality and retrospective in nature. The majority of the data does not reflect the treatment algorithms of today, with the introduction of focused radiation therapies and endoscopic surgical approaches. Changes to treatment algorithms have not been reflected in head-to-head studies examining the functional outcomes. The outcome measures reported have significant heterogeneity in the current literature, which limits the ability to draw definitive conclusions.

Moving forward, we propose that aspiration, intractable aspiration intervention, permanent gastrostomy tube requirements, and objective quality of life scales as objective measures that should be included in future research on this topic. Future studies and research should include well designed prospective trials with rigorous reporting of outcome measures.

Figure 1 Illustrates the PRISMA (Preferred Reporting Items for Systematic Reviews and Meta-Analyses) flow 


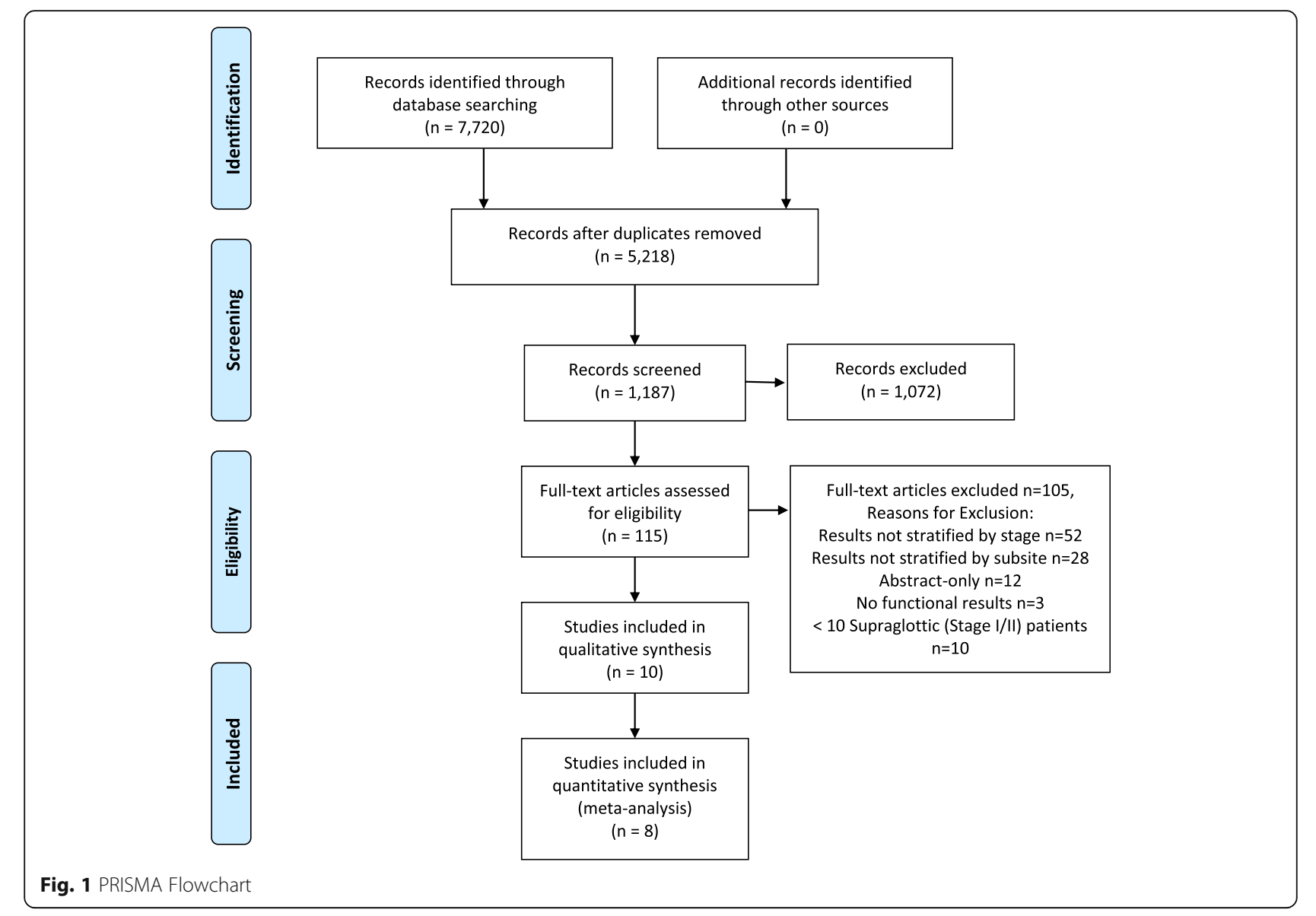

chart to identify the appropriate studies. Kappa statistic for the agreement at the abstract screening stage was 0.61 (95\% CI 0.37-0.85) indicating moderate agreement.

\section{Study characteristics and methodologic quality}

There were no randomized controlled trials comparing the functional outcomes of primary surgery versus radiation. Of the 10 studies included in the analysis, 6 were retrospective cohort studies, 4 were case series. There were no prospective studies included. There were 320 patients treated with OPS, including open partial laryngectomy, transoral laser microsurgery (TLM) or transoral robotic surgery (TORS). For the RT arm, there were 320 patients. Altogether, 640 patients were analyzed from 10 studies. There were 6 patients with early SSCC where the treatment modality was not specified and could not be included in the analyses. Characteristics of the included studies are summarized in Table 1. Overall, the quality of the included studies was moderate, this was for a variety of reasons, including no reference group (surgery or radiation arm only), short or unreported length of follow up, and unaccounted for patients. Table 2 summarizes the quality of the individual studies.

\section{Aspiration}

Aspiration was deemed a primary functional outcome measure of assessment. It was reported in 5 studies [11, 14, 19-21]. In one paper, this was evaluated under direct visualization with a functional endoscopic examination of swallowing, where the authors documented liquid penetration. The remainder of the papers reported patients with clinically evident late aspiration, and sequelae such as aspiration pneumonia. In the surgical arm, 9 out of 252 patients were reported to experience aspiration, for a pooled aspiration rate of $3.7 \%$ (95\% CI 1.9-6.9\%) (Fig. 2). In the RT arm, aspiration is reported in 15 out of 198 patients, for a pooled aspiration rate of $14.5 \%$ (95\% CI 9.1-22.5\%) (Fig. 3). The odds ratio is 1.23 (95\% CI 0.14-10.86) (Fig. 4).

\section{Intractable aspiration management}

In many of the studies, rate of functional laryngectomy and permanent tracheostomy dependence was reported. These statistics included some, but not all, of the patients who experienced late complications associated with aspiration events. This was reported in 5 studies, accounting for 186 surgical patients and 198 radiation patients. The pooled event rate for intractable aspiration 
Table 1 Study Characteristics

\begin{tabular}{|c|c|c|c|c|c|c|}
\hline Study ID & Location & Dates of Accrual & Age Mean (range) & OPS & RT & Stage \\
\hline Ambrosch 2018 & Italy & $2002-2012$ & Median $^{c} 62(33-88)$ & 27 & 0 & $\begin{array}{l}\text { T1 N0 }(n=8) \\
\text { T2 N0 }(n=19)\end{array}$ \\
\hline Bhattacharyya 2014 & India & $2006-2009$ & Median` $^{\complement} 59(31-80)$ & 0 & 10 & $\begin{array}{l}\text { T1 NO }(n=1) \\
\text { T2 NO }(n=9)\end{array}$ \\
\hline Carta 2018 & Italy & 2010-2017 & Mean $61.8(43-84)$ & 27 & 0 & $\begin{array}{l}\text { T1 N0 }(n=11) \\
\text { T2 NO }(n=16)\end{array}$ \\
\hline Chiesa Estomba 2015 & Spain & 2009-2012 & Mean 64 (45-88) & 72 & 0 & $\begin{array}{l}\text { T1 No }(n=44) \\
\text { T2 No }(n=28)\end{array}$ \\
\hline Chun 2010 & South Korea & 1991-2005 & Mean 65.5 & 25 & 10 & $\begin{array}{l}\text { T1 No }(n=14) \\
\text { T2 NO }(n=21)\end{array}$ \\
\hline Karatzanis 2010 & Germany & 1970-2004 & Mean 60 (36-83) & 78 & 0 & $\begin{array}{l}\text { T1 No }(n=29) \\
\text { T2 NO }(n=49)\end{array}$ \\
\hline Mendenhall 1996 & USA & 1964-1992 & NR & 0 & 99 & $\begin{array}{l}\mathrm{T}^{\mathrm{b}}(n=18) \\
\mathrm{T} 2(n=81)\end{array}$ \\
\hline Oridate 2009 & Japan & $2006-2007$ & Median $^{c} 76(45-90)$ & $N R^{a}$ & 11 & T2 No $(n=11)$ \\
\hline Orus 2000 & Spain & 1984-1996 & Mean 60.5 & 25 & 90 & $\begin{array}{l}\text { T1 N0 }(n=38) \\
\text { T2 NO }(n=77)\end{array}$ \\
\hline Spriano 1997 & Italy & 1983-1992 & NR & 66 & 100 & $\begin{array}{l}\text { T1 NO }(n=112) \\
\text { T2 No }(n=54)\end{array}$ \\
\hline
\end{tabular}

${ }^{\mathrm{a}} 6$ patients not accounted for in the results, ${ }^{\mathrm{b}}$ nodal staging not reported, ${ }^{\mathrm{c}}$ Median age presented

management in the surgical arm is $2.6 \%$ (95\% CI 1.0 6.8\%) (Fig. 5). The pooled event rate for the RT arm is $16.8 \%$ (95\% CI 10.8-25.0\%) (Fig. 6). The odds ratio comparing the two groups is $1.14(95 \%$ CI $0.04-33.45)$ (Fig. 7).

Permanent gastrostomy tube and swallowing dysfunction The rate of permanent gastrostomy tube was reported in four studies for the OPS group. This accounted for 198 patients. This outcome measure was not reported for the radiation group in any of the studies. The pooled event ratio for permanent gastrostomy tube dependence is $5.3 \%$ (95\% CI 2.6-10.5\%) (Fig. 8).

Table 2 Newcastle-Ottawa quality assessment scale for cohort studies

\begin{tabular}{|c|c|c|c|c|}
\hline Study & Selection & Comparability & Outcomes & Total Score \\
\hline Ambrosch $2018^{e}$ & 3 & 2 & 3 & 8 \\
\hline Bhattacharyya 2014 & 3 & 2 & 3 & 8 \\
\hline Carta 2018 & 2 & 1 & 3 & 6 \\
\hline Chiesa Estomba 2015 & 3 & 2 & 3 & 8 \\
\hline Chun $2010^{a}$ & 4 & 1 & 2 & 7 \\
\hline Karatzanis $2010^{\mathrm{b}}$ & 3 & 2 & 3 & 8 \\
\hline Mendenhall $1996^{c}$ & 3 & 0 & 1 & 4 \\
\hline Oridate $2009^{c, d}$ & 4 & 0 & 1 & 5 \\
\hline Orus $2000^{d}$ & 4 & 1 & 1 & 6 \\
\hline Spriano 1997 & 4 & 2 & 3 & 9 \\
\hline
\end{tabular}

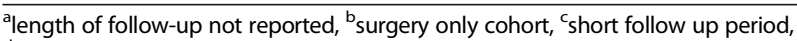

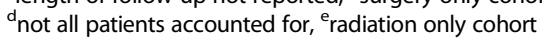

Additionally, Chun et al. compared OPS to RT with respect to functional outcomes of swallowing and speech. To evaluate swallowing, patients underwent a functional endoscopic examination of swallowing (FEES) using videoesophagofluoroscopy. The researchers looked specifically for evidence of aspiration. Liquid aspiration was identified $6 \%$ of patients in the OPS group $(n=1)$. There was no identified liquid aspiration in the RT cohort [11].

\section{Quality of life and voice outcomes}

Quality of life data was reported in one study. Another study reported objective voice outcome measures. Oridate et al. compared VRQOL, VHI-10, and GRBAS scores for T2 N0 SSCC against T1a, T1b, and T2 N0 glottic carcinomas and found no significant differences in functional outcomes [22].

Chun et al. used stroboscopy and acoustic waveform analysis to objectively evaluate voice outcomes. Abnormalities were in mucosal waveforms were identified $13 \%$ of the OPS cohort $(n=2)$ and $20 \%$ of the RT group $(n=2)$. These findings were not statistically significant [11].

\section{Discussion}

To our knowledge, this is the first systematic review evaluating the functional outcomes of surgery versus radiotherapy for early-stage SSCC. All studies that met inclusion criteria were retrospective in design and there were 3 head-to-head comparisons of surgery versus radiation. Aspiration events, management of intractable aspiration, and permanent gastrostomy tubes are some of the major 


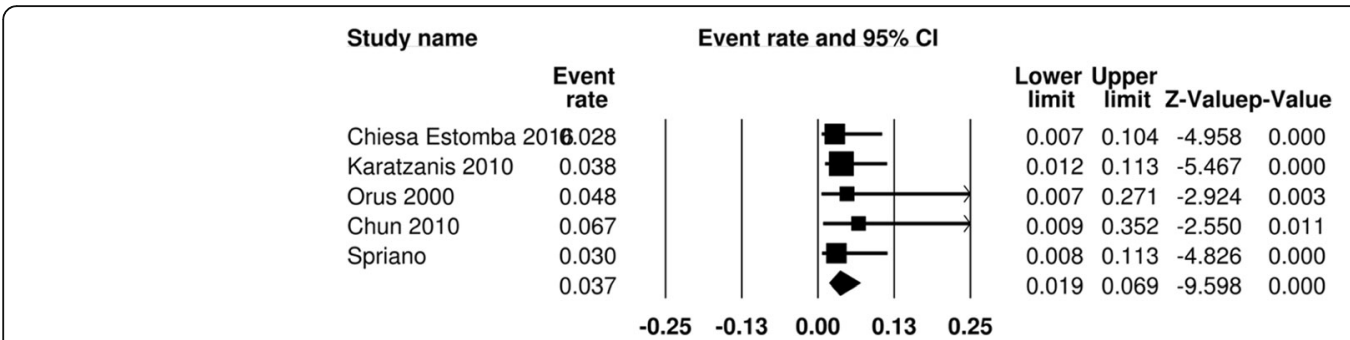

Fig. 2 Pooled event rate of aspiration for early stage SSCC treated with organ preservation surgery

indications of laryngeal function. There was insufficient data to thoroughly meta-analyze the two modalities with respect to these outcome measures. Furthermore, objective voice outcomes were available in just two studies. Quality of life measures were only found in one included paper.

Aspiration events were reported, in many cases, with little additional information. In some studies, these were clinically evident respiratory events, such as aspiration pneumonias that occurred late in the post-operative course, others reported only the number patients that experienced aspiration. Aspiration events are reported in $50 \%$ of studies $(n=5), 2$ of which were head-to-head comparisons.

For intractable aspiration intervention, there was data from both the surgical and radiation cohorts from multiple studies. This was reported as permanent tracheostomy dependence or conversion to a functional total laryngectomy. We calculated pooled event rates as well as pooled odds ratio. From the current data available, there is no strong evidence to suggest there is a difference between the two modalities with respect to this outcome measure.

With respect to permanent gastrostomy tube rate, there was no available data for the radiation cohort. Four papers reported this outcome measure for the surgical group ( $n=198$ patients). As a result, we were unable to make comparisons across the two treatment modalities.

Subjective and objective measures of voice outcome were sparsely and inconsistently reported. Quality of life measures were found in just one study, which actually compared T2 N0 supraglottic cancers to early glottic cancers.

\section{Surgery and radiotherapy for early stage SSCC}

Several factors are important in considering treatment options for patients with early laryngeal cancer. Survival outcomes are obviously at the forefront. While there is no RCT data comparing survival outcomes of patients with early SSCC, a 2016 meta-analysis by Patel et al. studying early stage SSCC suggests that patients who undergo primary surgery have better survival than those who underwent primary radiotherapy [8]. Laryngectomy rate is another important consideration, as most patients with early stage laryngeal cancer are candidates for organ preservation treatment. Finally, in the presence of equivalent survival for two treatment modalities, functional outcomes are important to assess in comparing treatment options.

Over the last 40 years the treatment of early stage laryngeal cancer has evolved [23, 24]. Surgery, in the form of open partial laryngectomy, was initially popular however many patients had poor functional outcomes including aspiration and impaired base of tongue and laryngeal movements leading to swallowing dysfunction [25]. Radiation then took over as the primary treatment modality for early stage laryngeal cancer, with the goal of mitigating many of the functional problems associated with open partial laryngectomy. However, radiation is not without its own side effects. In addition, minimally invasive surgical techniques including TORS and TLM are increasingly being used for early stage SSCC [23, 24], raising the question of whether the functional outcomes with TORS and TLM may be better than radiation. Unfortunately, there are very few studies reporting the functional outcomes of TORS and TLM for early stage supraglottic cancer.

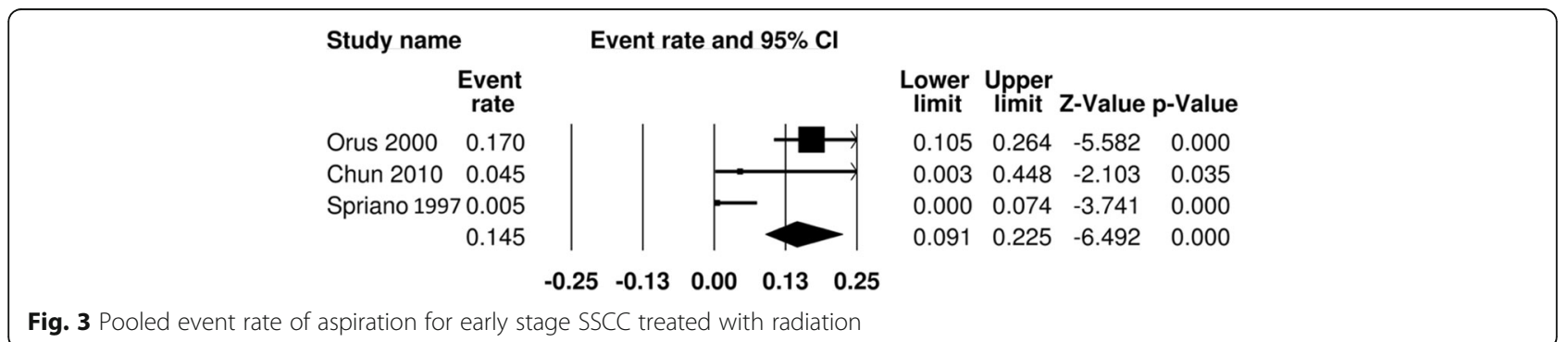




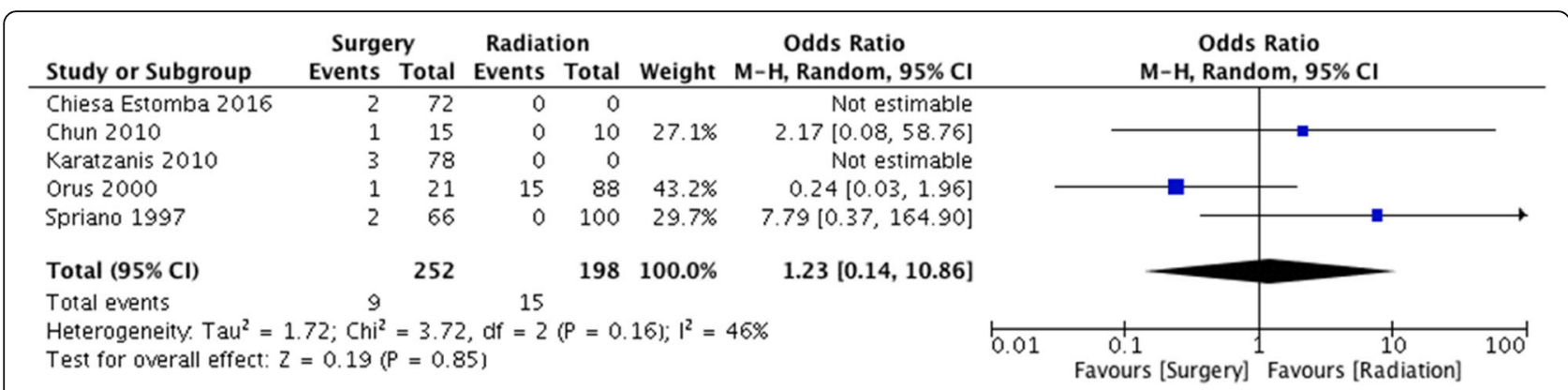

Fig. 4 Forest Plot of comparison between organ preservation surgery and radiation with respect to aspiration events

There are several advantages of RT. It preserves the laryngeal structures, it is generally well tolerated by patients, and increases surgical exposure to achieve excellent oncologic outcomes [26-29]. Radiation provides an effective treatment modality for patients not considered candidates for OPS due to their comorbid medical conditions. One of the disadvantages in treating early stage SSCC with RT, is that these patients are at high risk for developing a second primary and local regional recurrence $[5,6,30]$. If radiation is used as the primary treatment modality, most patients can only be salvaged with surgery, and, in the case of recurrent or new laryngeal cancer, the treatment is almost always total laryngectomy.

Surgical approaches include open surgery or transoral surgical approaches, including laser (TLM) and robotic (TORS). Organ-preserving surgery, both open and endoscopic approaches, offer several advantages over RT. As mentioned, patients with SSCC have reasonable 5-year overall survival rates, albeit with an increased risk of developing second primary aerodigestive tract malignancy $[5,6,30]$. Surgery can therefore be utilized as the first line, and, in the setting of recurrence, radiation can be used as salvage therapy. An additional advantage of surgery is the cost benefit of surgical intervention over radiotherapy $[31,32]$. Furthermore, surgical management with a neck dissection provides the opportunity to identify occult metastasis; an important consideration given that up to $30 \%$ patients with SSCC may have occult nodal metastasis [32]. Identification of occult metastasis allows for accurate staging of patients, and subsequently treatment with multimodality therapies.
Dombree et al. analyzed the cost of open supraglottic laryngectomy, TLM, and TORS in a Belgian model. Their study suggests the cost of open supraglottic laryngectomy similar to that of TLM in upfront surgical costs [31]. TORS tends to be more expensive primarily due to purchase and maintenance costs [31]. This study did not account for in-hospital costs such as length of admission, complications or readmission rates. With regard to glottic cancers, a cost analysis was carried out for a Canadian model comparing TLM to radiotherapy. This showed TLM to be a more cost-effective treatment option [33].

There are also disadvantages of surgery, including risk of general anesthetic, particularly in patients with comorbidities, bleeding, and infection. Pharyngocutaneous fistula, dysphagia and permanent tracheostomy dependence are specific risks of supraglottic laryngectomies. A criticism of OPS are the associated poor functional outcomes [25]. However, newer surgical techniques including TLM and TORS have gained popularity recently, and hold several advantages over open surgery and RT. In one study, TLM was compared to open surgery, resulting in reduced incidence of permanent gastrostomies and tracheostomies [20]. Since the introduction of TLM by Strong and Jako, there have been several reports investigating the role of TLM for supraglottic laryngectomy [34-48]. With respect to TORS, the majority of the studies report on all stages of supraglottic SCC $[20,43,48-$ 59]. With that in mind, long-term tracheostomy and gastric feeding tube rates range from 0 to $20 \%$ in patients treated with TORS $[49,52,55]$. None of the studies

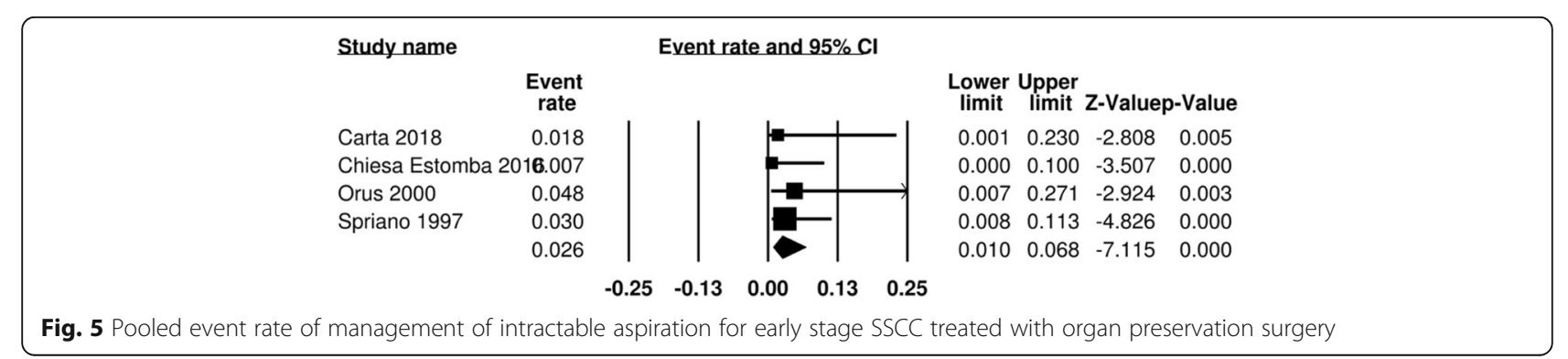




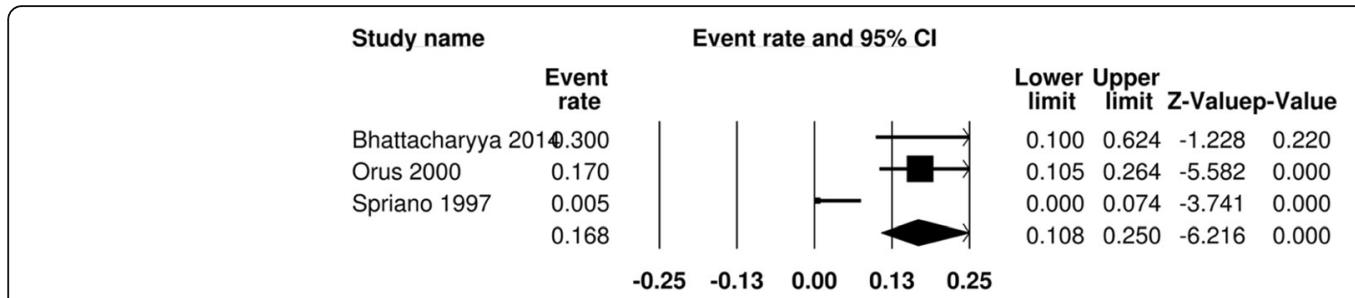

Fig. 6 Pooled event rate of definitive aspiration management for early stage SSCC treated with radiation

included in this systematic review examined the functional outcomes of TORS.

\section{Strengths}

There are several strengths to this review. To our knowledge, this is the first comprehensive review of all available literature comparing functional outcomes between surgery versus radiation for patients with early stage SSCC. It was designed, conducted and reported in accordance with published guidelines (PRISMA) and the study protocol, as well as search strategy, was outlined a priori. A comprehensive search strategy was undertaken and led to the review of 5218 unique citations of which ten studies met our inclusion criteria. This resulted in the analysis of a large number of patients with early stage SSCC $(n=640)$.

\section{Limitations}

As with all systematic reviews, the strength of the conclusions that can be drawn from this study depend on the quality of the primary studies. The included studies were evaluated with the Newcastle-Ottawa Scale for Assessing Cohort Studies, most of which were of moderate in overall quality. Next, although we only included studies published from 1990 forward, some of the studies in our review included patients treated well before that time period. This broad recruitment period includes many patients where treatment algorithms may not reflect today's standards. Given the improvements in medical imaging, some patients may have had regional nodal disease which was not evident on the available scans, therefore reflecting more advanced disease. Furthermore, many of the current treatment options, such intensity modulated radiotherapy (IMRT), TLM, and TORS were not in clinical practice prior to 1990. The types of radiation and protocols used were not clearly outlined in the studies included. As well, given the broad recruitment periods for some of these studies, different radiotherapy protocols would have been offered to the patients according to the available therapies at that time.

All 10 studies that met the inclusion criteria were retrospective study designs and there were no randomized controlled trials. Retrospective studies have inherent biases including selection biases. Patient's with medical comorbidities may not have been deemed appropriate surgical candidates and only offered radiotherapy, which may not be reflected in the results.

Significant heterogeneity was noted between the outcome measures of the included studies. In the surgical group, not all patients may have received the same type or extent of surgery, including elective neck dissections, TLM, and TORS operations. We only considered English language studies for our systematic review, which limited the number of titles screened and studies included, however, the effect of this would likely be small.

The heterogeneity of the functional outcomes reported limited our ability to meta-analyze the data. Additionally, many of the outcomes were sparsely reported or reported for only one of the arms of study, either OPS or RT. The overall paucity of data limits our ability to draw conclusions.

Given the lack of high level evidence guiding the optimal management of early stage supraglottic cancer and potential biases of retrospective studies, a head to head comparison between newer modalities such as TLM and

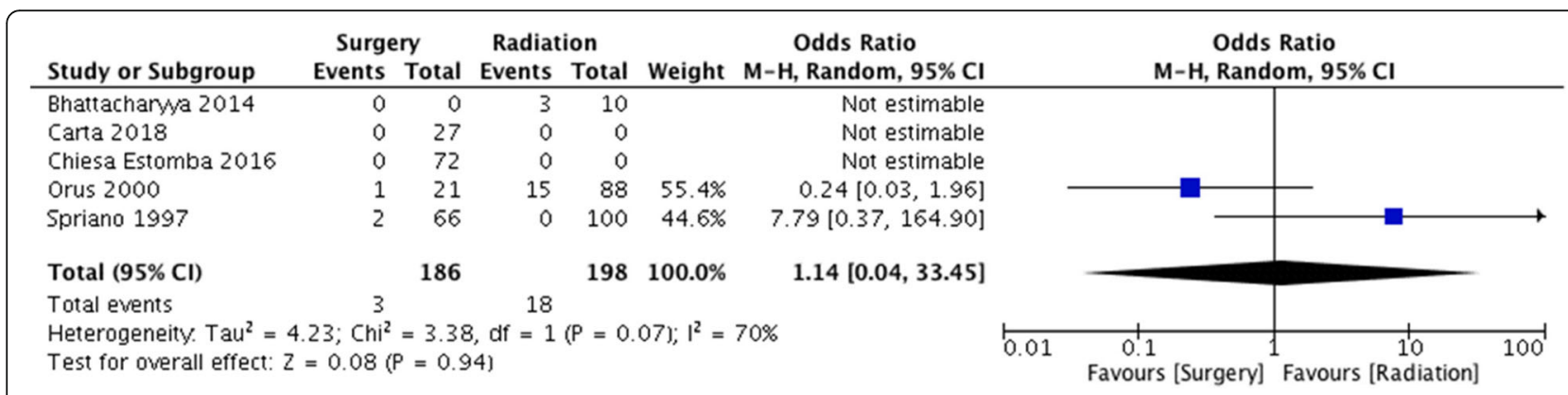

Fig. 7 Forest Plot of comparison between organ preservation surgery and radiation with respect to management of intractable aspiration with permanent tracheostomy or functional laryngectomy 


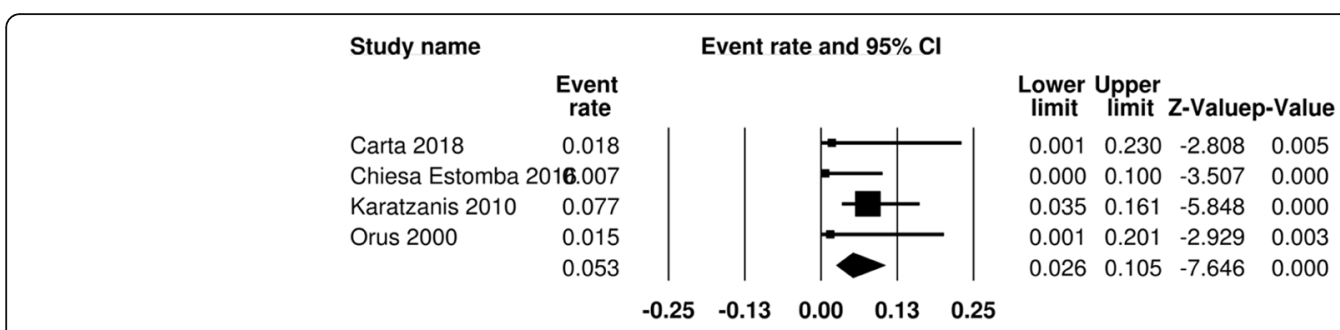

Fig. 8 Pooled event rate of permanent gastrostomy tube for early stage SSCC treated with organ preservation surgery

TORS with RT is critical in determining the therapeutic algorithm that can yield better functional outcomes in early stage SSCC patient. Many studies were ruled out as part of our protocol due to the stratification of results with respect to laryngeal subsite and staging criteria (including advanced stage disease). The method in which functional results are reported, is often less rigorous than the reporting of survival and locoregional control. The use of objective measures and validated tools was limited and not consistent across studies.

Traditionally, studies comparing surgery and radiation have been challenging to accrue patients to. Ongoing efforts comparing OPS to RT for oropharyngeal cancer are underway and actively accruing $[60,61]$, demonstrating that a head-to-head comparison of surgery and radiation is a possibility for patients with early stage supraglottic cancer. High level of evidence is important in the development of treatment guidelines for patients with early stage disease that have a surprisingly poor prognosis, compared to other early stage head and neck cancers. Traditionally, OPS has been seen as an option with poor functional outcomes. We did not find evidence to support one modality being better than another with respect to functional outcomes.

\section{Conclusions}

There is an overall paucity of literature available regarding functional outcomes in the treatment of early stage SSCC patients, the majority of which is moderate in overall quality and retrospective in nature. The majority of the data does not reflect the treatment algorithms of today, with the introduction of focused radiation therapies and endoscopic surgical approaches. Changes to treatment algorithms have not been reflected in head-to-head studies examining the functional outcomes. The outcome measures reported have significant heterogeneity in the current literature, which limits the ability to draw definitive conclusions.

Moving forward, we propose that aspiration, intractable aspiration intervention, permanent gastrostomy tube requirements, and objective quality of life scales as objective measures that should be included in future research on this topic. Future studies and research should include well designed prospective trials with rigorous reporting of outcome measures.

\begin{abstract}
Abbreviations
Cl: Confidence Intervals; FEES: Functional endoscopic examination of swallowing; NCCN: National comprehensive cancer network; ND: Neck dissection; NR: Not reported; OPS: Organ preservation surgery; OR: Odds Ratios; PRISMA: Preferred Reporting Items for Systematic Reviews and Meta-Analyses; RT: Radiation Therapy; SSCC: Supraglottic squamous cell carcinoma;

TLM: Transoral endoscopic laser microsurgery; TORS: Transoral robotic surgery
\end{abstract}

\section{Acknowledgements}

The authors would like to acknowledge Alla lansavitchene at London Health Sciences for her help with designing the search strategy for literature review.

Funding

No funding was required or made available. The study was presented at the Canadian Society of Otolaryngology Meeting in Quebec City, QC.

Availability of data and materials

Literature review as well as data analysis can be made available on request.

\section{Author's contributions}

BVW, KBP and SDM - Designed study design, search strategy, title screen, abstract screen, data extraction, data analysis, review and writing of the manuscript. ACN, KF, JY - review and writing of the manuscript. All authors read and approved the final manuscript.

\section{Authors' information}

BWW - PGY 2 Resident, Otolaryngology - Head and Neck Surgery, Western University, London, Ontario.

KBP - Head and Neck Oncology and Reconstructive Fellow, Otolaryngology - Head and Neck Surgery, The Ohio State University, Columbus, OH. ACN - Associate Professor, Otolaryngology - Head and Neck Surgery, Western University, London, Ontario.

KF - Professor, Otolaryngology - Head and Neck Surgery, Western University, London, Ontario.

JY - Professor, Otolaryngology - Head and Neck Surgery, Western University, London, Ontario.

SDM - Assistant Professor, Otolaryngology - Head and Neck Surgery,

Western University, London, Ontario.

Ethics approval and consent to participate

Not Applicable. This was a systematic review and meta-analysis.

Consent for publication

Not applicable. No patients were directly involved. No consent for publication is required.

Competing interests

No competing interests to report.

\section{Publisher's Note}

Springer Nature remains neutral with regard to jurisdictional claims in published maps and institutional affiliations.

\section{Author details}

'Department of Otolaryngology, Head \& Neck Surgery, Schulich Medicine \& Dentistry, Western University, London Health Sciences Centre, Victoria 
Hospital, London, Ontario, Canada. ²Department of Otolaryngology, Head and Neck Surgery, The Ohio State University, Columbus, OH, USA.

\section{Received: 8 April 2018 Accepted: 22 November 2018 Published online: 18 December 2018}

\section{References}

1. Edge S BD, Compton C, Fritz A, Greene F, Trotti A. AJCC cancer staging handbook. Springer-Verlag New York; 2010.

2. Hoffman HT, Porter K, Karnell LH, et al. Laryngeal cancer in the United States: changes in demographics, patterns of care, and survival. Laryngoscope. 2006;116:1-13.

3. Am Cancer Soc Key Statistics for Laryngeal and Hypopharyngeal Cancers. 2018. American Cancer Society; 2018. Available at: https:/www.cancer.org/ cancer/laryngeal-and-hypopharyngeal-cancer/about/key-statistics.html.

4. NCCN clinical practice guidelines in oncology in Head and Neck Cancers (version 1.2015). http://www.nccn.org/professionals/physician_gls/pdf/headand-neck.pdf (2015). Accessed 10 Nov 2015.

5. Piccirillo F. Cancer of the larynx in SEER survival monograph: cancer survival among adults: U.S. SEER Program 1988-2001, Patient and Tumor Characteristics. National Cancer Insitutite, SEER Program. NIH Pub. No, 076215, Bethesda, MD, 2007.

6. Cosetti M, Yu GP, Schantz SP. Five-year survival rates and time trends of laryngeal cancer in the US population. Arch Otolaryngol. 2008;134:370-9.

7. Chen AY, Fedewa S, Zhu J. Temporal trends in the treatment of early- and advanced-stage laryngeal cancer in the United States, 1985-2007. Arch Otolaryngol. 2011;137:1017-24.

8. Arshad H, Jayaprakash V, Gupta V, et al. Survival differences between organ preservation surgery and definitive radiotherapy in early supraglottic squamous cell carcinoma. Otolaryngol Head Neck Surg. 2014;150:237-44.

9. Patel KB, Nichols AC, Fung K, Yoo J, MacNeil SD. Treatment of early stage Supraglottic squamous cell carcinoma: meta-analysis comparing primary surgery versus primary radiotherapy. J Otolaryngol Head Neck Surg. 2018:47:19.

10. Bron $L P$, Soldati $D$, Zouhair $A$, et al. Treatment of early stage squamous-cell carcinoma of the glottic larynx: endoscopic surgery or cricohyoidoepiglottopexy versus radiotherapy. Head Neck. 2001;23:823-9.

11. Chun JY, Kim YH, Choi EC, Byeon HK, Jung J, Kim SH. The oncologic safety and functional preservation of supraglottic partial laryngectomy. Am J Otolaryngol. 2010;31:246-51.

12. Jones AS, Fish B, Fenton JE, Husband DJ. The treatment of early laryngeal cancers (T1-T2 NO): surgery or irradiation? Head Neck. 2004;26:127-35.

13. Laccourreye $\mathrm{O}$, Weinstein $\mathrm{G}$, Chabardes $\mathrm{E}$, Housset M, Laccourreye H, Brasnu D. T1 squamous cell carcinoma of the arytenoid. Laryngoscope. 1992;102: 896-900.

14. Orus $C$, Leon $X$, Vega $M$, Quer $M$. Initial treatment of the early stages $(I, I)$ of supraglottic squamous cell carcinoma: partial laryngectomy versus radiotherapy. Eur Arch Otorhinolaryngol. 2000:257:512-6.

15. Santos CR, Kowalski LP, Magrin J, et al. Prognostic factors in supraglottic carcinoma patients treated by surgery or radiotherapy. Ann Otol Rhinol Laryngol. 1998;107:697-702.

16. Sessions DG, Lenox J, Spector GJ. Supraglottic laryngeal cancer: analysis of treatment results. Laryngoscope. 2005;115:1402-10.

17. Spector JG, Sessions DG, Emami B, Simpson J, Haughey B, Fredrickson JM. Squamous cell carcinomas of the aryepiglottic fold: therapeutic results and long-term follow-up. Laryngoscope. 1995;105:734-46.

18. Newcastle-Ottawa Scale [type]. Updated http://www.ohri.ca/programs/ clinical_epidemiology/oxford.htm.

19. Spriano G, Antognoni P, Piantanida R, et al. Conservative management of T1-T2N0 supraglottic cancer: a retrospective study. Am J Otolaryngol. 1997; 18:299-305.

20. Karatzanis AD, Psychogios G, Zenk J, et al. Evaluation of available surgical management options for early supraglottic cancer. Head Neck. 2010;32: 1048-55.

21. Chiesa Estomba CM, Reinoso FA, Velasquez AO, Fernandez JL, Conde JL, Hidalgo CS. Complications in CO2 laser Transoral microsurgery for larynx carcinomas. International archives of otorhinolaryngology. 2016;20:151-5.

22. Oridate N, Homma A, Suzuki S, Nakamaru Y, Suzuki F, Hatakeyama H, Fukuda S. Voice-related quality of life after treatment of laryngeal cancer Archives of Otolaryngology-Head \& Neck Surgery 2009: 135(4): 363-368.
23. De Almeida JR, Byrd JK, Wu R, Stucken CL, Duwuri U, Goldstein DP, et al. A systematic review of transoral robotic surgery and radiotherapy for early oropharynx cancer: a systematic review. Laryngoscope. 2014;124(9):2096-102.

24. von Scotti F, Kapsreiter M, Scherl C, Iro H, Bohr C. A 9-year analysis of transoral laser microsurgery (TLM) of head and neck cancer on their potential suitability for transoral robotic surgery (TORS) for estimation of future TORS-specific caseload. Eur Rev Med Pharmacol Sci. 2018;22:2949-53.

25. Lewin JS, Hutcheson KA, Barringer DAet al. Functional analysis of swallowing outcomes after supracricoid partial laryngectomy. Head Neck 2008; 30:559-566.

26. Kerr P, Mark Taylor S, Rigby Met al. Oncologic and voice outcomes after treatment of early glottic cancer: transoral laser microsurgery versus radiotherapy. J Otolaryngol Head Neck Surg 2012; 41:381-388.

27. Spielmann PM, Majumdar S, Morton RP. Quality of life and functional outcomes in the management of early glottic carcinoma: a systematic review of studies comparing radiotherapy and transoral laser microsurgery. Clin Otolaryngol. 2010;35:373-82.

28. Kennedy JT, Paddle PM, Cook BJ, Chapman P, Iseli TA. Voice outcomes following transoral laser microsurgery for early glottic squamous cell carcinoma. J Laryngol Otol. 2007;121:1184-8.

29. Mendenhall WM, Parsons JT, Mancuso AA, Stringer SP, Cassisi NJ. Radiotherapy for squamous cell carcinoma of the Supraglottic larynx: an alternative to surgery. Head Neck. 1996;18:24-35.

30. Vaamonde P, Martin C, del Rio M, LaBella T. Second primary malignancies in patients with cancer of the head and neck. Otolaryngol Head Neck Surg. 2003;129:65-70

31. Dombree M, Crott R, Lawson G, Janne P, Castiaux A, Krug B. Cost comparison of open approach, transoral laser microsurgery and transoral robotic surgery for partial and total laryngectomies. Eur Arch Otorhinolaryngol. 2014;271:2825-34.

32. Byers RM, Wolf PF, Ballantyne AJ. Rationale for elective modified neck dissection. Head Neck Surg. 1988;10:160-7.

33. Phillips TJ, Sader C, Brown T, et al. Transoral laser microsurgery versus radiation therapy for early glottic cancer in Canada: cost analysis. J Otolaryngol Head Neck Surg. 2009;38:619-23.

34. Strong MS, Jako GJ. Laser surgery in the larynx. Early clinical experience with continuous CO 2 laser. Ann Otol Rhinol Laryngol. 1972;81:791-8.

35. Breda E, Catarino R, Monteiro E. Transoral laser microsurgery for lanyngeal carcinoma: survival analysis in a hospital-based population. Head Neck. 2015;37:1181-6.

36. Bussu F, Almadori G, De Corso E, et al. Endoscopic horizontal partial laryngectomy by $\mathrm{CO}(2)$ laser in the management of supraglottic squamous cell carcinoma. Head Neck. 2009:31:1196-206.

37. Canis M, Martin A, lhler F, et al. Results of transoral laser microsurgery for supraglottic carcinoma in 277 patients. Eur Arch Otorhinolaryngol. 2013;270: 2315-26.

38. Csanady M, Czigner J, Vass G, Jori J. Transoral CO2 laser management for selected supraglottic tumors and neck dissection. Eur Arch Otorhinolaryngol. 2011;268:1181-6.

39. Davis RK, Kriskovich MD, Galloway EB 3rd, Buntin CS, Jepsen MC. Endoscopic supraglottic laryngectomy with postoperative irradiation. Ann Otol Rhinol Laryngol. 2004;113:132-8.

40. Grant DG, Salassa JR, Hinni ML, Pearson BW, Hayden RE, Perry WC. Transoral laser microsurgery for carcinoma of the supraglottic larynx. Otolaryngol Head Neck Surg. 2007;136:900-6.

41. Iro H, Waldfahrer F, Altendorf-Hofmann A, Weidenbecher M, Sauer R, Steiner W. Transoral laser surgery of supraglottic cancer: follow-up of 141 patients. Arch Otolanyngol Head Neck Surg. 1998;124:1245-50.

42. Peretti G, Piazza C, Ansarin M, et al. Transoral CO2 laser microsurgery for tisT3 supraglottic squamous cell carcinomas. Eur Arch Otorhinolaryngol. 2010; 267:1735-42.

43. Solares CA, Strome M. Transoral robot-assisted CO2 laser supraglottic lanyngectomy: experimental and clinical data. Laryngoscope. 2007;117:817-20.

44. Vilaseca I, Blanch JL, Berenguer J, et al. Transoral laser microsurgery for locally advanced (T3-T4a) supraglottic squamous cell carcinoma: sixteen years of experience. Head Neck. 2016;38:1050-7.

45. Cabanillas R, Rodrigo JP, Llorente JL, Suarez C. Oncologic outcomes of transoral laser surgery of supraglottic carcinoma compared with a transcervical approach. Head Neck. 2008;30:750-5.

46. Cabanillas R, Rodrigo JP, Llorente JL, Suarez V, Ortega P, Suarez C. Functional outcomes of transoral laser surgery of supraglottic carcinoma compared with a transcervical approach. Head Neck. 2004;26:653-9. 
47. Kollisch M, Werner JA, Lippert BM, Rudert H. Functional results following partial supraglottic resection. Comparison of conventional surgery vs. transoral laser microsurgery. Adv Otorhinolaryngol. 1995;49:237-40.

48. More YI, Tsue TT, Girod DA, et al. Functional swallowing outcomes following transoral robotic surgery vs primary chemoradiotherapy in patients with advanced-stage oropharynx and supraglottis cancers. JAMA Otolaryngol Head Neck Surg. 2013;139:43-8.

49. Razafindranaly V, Lallemant B, Aubry K, et al. Clinical outcomes with transoral robotic surgery for supraglottic squamous cell carcinoma: experience of a French evaluation cooperative subgroup of GETTEC. Head Neck. 2016;38:E1097-101.

50. Weinstein GS, O'Malley BW Jr, Snyder W, Hockstein NG. Transoral robotic surgery: supraglottic partial laryngectomy. Ann Otol Rhinol Laryngol. 2007; 116:19-23.

51. Durmus K, Gokozan HN, Ozer E. Transoral robotic supraglottic laryngectomy: surgical considerations. Head Neck. 2015;37:125-6.

52. Ozer E, Alvarez B, Kakarala K, Durmus K, Teknos TN, Carrau RL. Clinical outcomes of transoral robotic supraglottic laryngectomy. Head Neck. 2013; 35:1158-61.

53. Park YM, Byeon HK, Chung HP, Choi EC, Kim SH. Comparison of treatment outcomes after transoral robotic surgery and supraglottic partial laryngectomy: our experience with seventeen and seventeen patients respectively. Clin Otolaryngol. 2013;38:270-4.

54. Park YM, Lee WJ, Lee JG, et al. Transoral robotic surgery (TORS) in laryngeal and hypopharyngeal cancer. J Laparoendosc Adv S. 2009;19:361-8.

55. Dowthwaite S, Nichols AC, Yoo J, et al. Transoral robotic total laryngectomy: report of 3 cases. Head Neck. 2013;35:E338-42.

56. Mendelsohn AH, Remacle M. Transoral robotic surgery for laryngeal cancer. Curr Opin Otolaryngol Head Neck Surg. 2015;23:148-52.

57. Mendelsohn AH, Remacle M, Van Der Vorst S, Bachy V, Lawson G. Outcomes following transoral robotic surgery: supraglottic laryngectomy. Laryngoscope. 2013;123:208-14.

58. Olsen SM, Moore EJ, Koch CA, Price DL, Kasperbauer JL, Olsen KD. Transoral robotic surgery for supraglottic squamous cell carcinoma. Am J Otolaryngol. 2012;33:379-84.

59. Rodrigo JP, Suarez C, Silver CE, et al. Transoral laser surgery for supraglottic cancer. Head Neck. 2008;30:658-66.

60. Nichols AC, Yoo J, Hammond JA, et al. Early-stage squamous cell carcinoma of the oropharynx: radiotherapy vs. trans-oral robotic surgery (ORATOR)-study protocol for a randomized phase II trial. BMC Cancer. 2013;13:133.

61. EORTC 1420-HNCG-ROG "The best of" trial. http://www.eortc.org/research_ field/clinical-detail/1420/

Ready to submit your research? Choose BMC and benefit from:

- fast, convenient online submission

- thorough peer review by experienced researchers in your field

- rapid publication on acceptance

- support for research data, including large and complex data types

- gold Open Access which fosters wider collaboration and increased citations

- maximum visibility for your research: over $100 \mathrm{M}$ website views per year

At BMC, research is always in progress.

Learn more biomedcentral.com/submissions 